\title{
Accurate localization of the symptomatic nerve roots of patients with adult degenerative scoliosis using diffusion tensor imaging
}

\section{Qian Wang}

The First Affiliated Hospital of Nanjing Medical University,

\section{Peng Gao}

The First Affiliated Hospital of Nanjing Medical University,

\section{Pengyu Tang}

The First Affiliated Hospital of Nanjing Medical University,

\section{Jian Chen}

The First Affiliated Hospital of Nanjing Medical University,

\section{Shujie Zhao}

The First Affiliated Hospital of Nanjing Medical University,

\section{Qingqing Li}

The First Affiliated Hospital of Nanjing Medical University,

\section{Wei Zhou}

The First Affiliated Hospital of Nanjing Medical University,

\section{Guoyong Yin ( $\sim$ guoyong_yin@sina.com )}

The First Affiliated Hospital of Nanjing Medical University,

Jin Fan

The First Affiliated Hospital of Nanjing Medical University,

\section{Research Article}

Keywords: adult degenerative scoliosis (ADS), MR diffusion tensor imaging (DTI), Japanese Orthopaedic Association (JOA), Oswestry Disability Index (ODI), Visual Analog Scale-Back Pain (VAS-BP), Visual Analog Scale-Leg Pain (VAS-LP)

Posted Date: May 12th, 2021

DOI: https://doi.org/10.21203/rs.3.rs-500269/v1

License: (c) (i) This work is licensed under a Creative Commons Attribution 4.0 International License. Read Full License 


\title{
Accurate localization of the symptomatic nerve roots of patients with adult degenerative scoliosis using diffusion tensor imaging
}

\author{
Qian Wang ${ }^{\dagger}$ Peng Gao', Pengyu Tang, Jian Chen, Shujie Zhao, Wei Zhou, Qingqing Li, \\ Guoyong Yin * and Jin Fan * \\ *Correspondence: guoyong_yin@sina.com; fanjin@njmu.edu.cn \\ tQian Wang and Peng Gao contributed equally to this work. \\ Department of Orthopedics, The First Affiliated Hospital of Nanjing Medical University, No. \\ 300 Guangzhou Road, Nanjing 210029, People's Republic of China
}

\section{Abstract}

Background: In the diagnosis and treatment of adult degenerative scoliosis (ADS), the localization of symptomatic nerve roots is mainly based on the symptoms and signs of patients. Because of spinal rotation deformity, conventional MRI cannot accurately select the level of intervertebral disc for scanning. In the present study, MR diffusion tensor imaging (DTI) was used to diagnose symptomatic nerve roots in patients with ADS, and its effectiveness was evaluated by comparing with diagnosis based on symptoms and signs of nerve root block. Methods: Thirty-six patients who were clinically diagnosed to have ADS underwent routine MRI and DTI examinations, and fractional anisotropy (FA) of each nerve root in the lumbar spine was quantitatively measured. The t-test was used to compare the FA values of nerve roots with and without neurological symptoms. Preoperative and postoperative clinical evaluations were conducted on the patients, and the t-test was used to evaluate the clinical effects of surgery.

Results: The FA values of symptomatic and asymptomatic nerve roots were significantly different $(P<0.01)$. The DTI findings of symptomatic nerve roots were consistent with the diagnosis based on symptoms and nerve root block. The Japanese Orthopaedic Association (JOA), Oswestry Disability Index (ODI), Visual Analog Scale-Back Pain (VAS-BP), and Visual Analog Scale-Leg Pain (VAS-LP) scores of the postoperative patients were significantly improved as compared to those in the preoperation stage; the Cobb, Lumbar Lordosis (LL), Pelvic Tilt (PT), and Sacral Slope (SS) angles were corrected significantly post operation.

Conclusion: The FA value can be used as a marker for predicting symptomatic nerve root and is consistent with clinical symptoms. According to the results of DTI, precise nerve root 
decompression can relieve the symptoms. Therefore, DTI can be used as an effective auxiliary method for symptomatic nerve root localization in patients with degenerative scoliosis.

\section{Introduction}

With the aggravation of population aging, the incidence of adult degenerative scoliosis (ADS) is also rising [1]. Unlike idiopathic scoliosis, degenerative scoliosis is often accompanied by lumbar degenerative symptoms. Therefore, for the treatment of patients with ADS, both scoliosis correction and symptomatic nerve root decompression are required to alleviate neurological symptoms [2]. Previously, the localization of symptomatic nerve roots was often based on symptoms, but this approach resulted in errors in correct localization [3]. In routine cases of lumbar degeneration, lumbar magnetic resonance imaging (MRI) is often used as an important imaging modality. However, for patients with ADS, because of spinal rotation deformity, MRI cannot accurately select the intervertebral disc level for scanning (Fig. 1), and normal MRI cannot perform functional imaging of nerves [4]. Therefore, a better imaging technology is needed to accurately localize the symptomatic nerve root, which is very important for the selection of nerve root decompression segment in patients with ADS.

MRI has been widely used as an imaging modality in spinal diseases, and the relatively new diffusion tensor imaging (DTI) can provide valuable quantitative data for peripheral nerves and can quantitatively evaluate the functional status of nerves [5]. DTI imaging can quantitatively reflect the pathological changes of peripheral nerves through fractional anisotropy (FA) and apparent diffusion coefficient (ADC) and can provide effective parameters to diagnose nerve root disease caused by lumbar degeneration [6]. Some studies have shown that the FA value is more accurate than the ADC value to evaluate the function of nerve root under compression [7]. DTI parameters are more sensitive and specific than those of conventional MR imaging in localizing symptomatic nerve roots [8]. Previous studies have also confirmed a close correlation between clinical symptoms and DTI parameters in patients with lumbar degenerative disease [9]. Previous reports of DTI included only single-level lumbar degenerative cases $[6,10]$, and there were no related reports on multilevel degenerative cases with degenerative scoliosis. For patients with multisegmental lumbar degenerative disease, the accurate localization of the symptomatic nerve root and minimization of the scope of surgery are helpful to yield satisfactory surgical results for patients [11]. Therefore, the main purpose of the present study is to determine 
the clinical significance of the use of DTI as an imaging technique to localize the responsible nerve root in patients with ADS.

\section{Methods}

This study was performed on 36 patients with degenerative scoliosis.

\section{Patient data}

The following inclusion criteria were used: patients with degenerative scoliosis with clinical manifestations of low back pain and nerve root symptoms, and patients showing Cobb angle $>20^{\circ}$ in imaging findings and requiring surgical treatment due to ineffective conservative treatment. The following exclusion criteria were considered: patients with a history of spinal surgery, infection, tumor, nervous system disease, etc. From June 2017 to September 2020, 36 patients (16 males, age $54.3 \pm 8.5$ years; 20 females, age $56.6 \pm 9.6$ years) with degenerative scoliosis underwent surgery in our hospital. Preoperative clinical evaluation, routine $\mathrm{X}$-ray, and CT, MRI, and DTI examinations were performed. Imaging was performed by imaging doctors with specialization in DTI. Clinical evaluation was performed at 1 week, 3 months, and 6 months after operation.

\section{Symptomatic nerve root localization}

First, the responsible nerve root was located according to the symptoms and signs. Sensory area localization: L3 nerve root, femoral condyle; L4 nerve root, malleolus; L5 nerve root, dorsalis pedis third metatarsophalangeal joint; S1 nerve root, lateral heel. Key muscles: L3 nerve root, quadriceps femoris; L4 nerve root, tibialis anterior; L5 nerve root, extensor longus; S1 nerve root, ankle plantar flexor [12]. For patients for whom the nerve could not be located according to the symptoms and signs, nerve root block was performed to identify the responsible nerve root. The method was the same as that reported by Yan Liang et al.[13]. Under fluoroscopy, $1 \mathrm{ml}$ of $1 \%$ lidocaine was injected through the intervertebral foramen (Fig. 2). If the patient's symptoms were relieved, the located nerve was considered to be the responsible nerve root; if there was no remission, the possibility of involvement of the nerve root was ruled out. According to clinical manifestations, nerve root block was performed by senior spine surgeons in our hospital. All patients underwent DTI examination, and the results of DTI were compared with the above results.

\section{Imaging analysis}

General MRI and DTI examinations were performed with a GE Discovery 3.0T MR scanner. 
FA and diffusion tractor tractography (DTT) were evaluated by a GE Advantage 4.6 workstation. FA values of L3 to S1 nerve roots were measured. Three regions of interest (ROIs) were selected for each nerve root, which were located in the subarticular area, foraminal area, and extraforaminal area. The results of DTT were used to confirm whether the nerve of the symptomatic side had abnormalities such as interruption, sparseness, and decreased hue. ROI location and DTT nerve root reconstruction were performed by two experienced radiologists. These radiologists were unaware of the study design and clinical data. The consistency of the results within and between groups was evaluated.

\section{Surgical method}

After general anesthesia, the patient assumed the prone position. A median incision was performed to expose the lamina and articular process. Transpedicular screws were inserted under fluoroscopy. According to the results of DTI, fenestration was performed at the responsible segment. Resection of the hyperplastic facet joint, hyperplastic ligamentum flavum, herniated intervertebral disc, and lateral recess and nerve root canal decompression were performed. The scoliosis was corrected gradually by placing a pre-bending bar. Segmental interbody fusion was performed after discectomy. Finally, the incision was sutured after a negative pressure flow tube was placed. All operations were performed by a spine surgeon with more than 20 years of experience.

\section{Clinical evaluation}

$\mathrm{X}$-ray examination was performed in all patients at 1 week, 3 months, and 6 months after operation to observe the correction of scoliosis, internal fixation position, and interbody fusion. The scores of Japanese Orthopaedic Association (JOA), Oswestry Disability Index

(ODI), Visual Analog Scale-Back Pain (VAS-BP), and Visual Analog Scale-Leg Pain (VAS-LP) were assessed at 1 week, 3 months, and 6 months after operation.

\section{Data analysis}

SPSS 25.0 software was used for statistical analysis. Statistical data were analyzed with Graphpad Prism 8.0. Descriptive statistics were presented as mean and standard deviation. The $t$-test was used to analyze the differences in the FA value between the symptomatic side and the asymptomatic side. The preoperative and postoperative Cobb, lumbar lordosis (LL), pelvic tilt (PT), and sacral slope (SS) angles and the JOA, ODI, VAS-BP, and VAS-LP scores were compared by the t-test. 


\section{Results}

\section{Preoperative evaluation results}

According to the symptoms and signs, the responsible nerve root could be accurately determined for 13 patients, and the nerve root could not be clearly localized for 23 patients and needed nerve root block. There were 13 patients with single segment symptoms, 21 patients with double segment symptoms, and 2 patients with three segment symptoms. There were 23 patients with unilateral symptoms and 13 patients with bilateral symptoms. The responsible nerve roots were L3 (6 patients), L4 (19 patients), L5 (25 patients), and S1 (11 patients) (Table 1).

\section{DTI results}

By referring to a previous study on FA values of normal nerve roots conducted in the Department of Radiology of our hospital [14], the nerve roots with lower FA values in the ROI area were regarded as symptomatic nerve roots. The FA values of symptomatic and asymptomatic nerve roots of each segment were compared. The average FA values of three $\mathrm{ROI}$ regions of each nerve root were as follows: L3: symptomatic $(0.162 \pm 0.026)$, asymptomatic (0.231 \pm 0.031); L4: symptomatic (0.145 \pm 0.024), asymptomatic $(0.254 \pm$ 0.032); L5: symptomatic (0.152 \pm 0.033), asymptomatic (0.237 \pm 0.024$)$; S1: symptomatic $(0.132 \pm 0.021)$, asymptomatic $(0.201 \pm 0.034)$. Significant differences were observed in FA values between symptomatic and asymptomatic L3-S1 nerve roots in the t-test (Table 2). The results were compared with the previous nerve root localization results, and the coincidence rate was 98.6\% (Table 1). DTT was performed in all patients. There were 24 patients with segmental sparsity, narrowing, and truncation of nerve root fiber tracts, which accounted for 92.3\% of the total cases (Fig. 3). These results were consistent with those of the FA value defining symptomatic nerve root.

\section{Surgical results}

Long segment fixation, decompression at the symptomatic nerve root, resection of the intervertebral disc segment, and bone graft fusion were performed in all patients. The operation time was $152.59 \pm 25.21 \mathrm{~min}$, the intraoperative blood loss was $598.56 \pm 156.32$ $\mathrm{ml}$, and the average hospital stay was $8.45 \pm 4.59$ days. Complications occurred in 2 patients (7.7\%). One patient with an infection of the incision was treated by increasing the dose of antibiotics and changing the wound dressing. In the other patient, dural rupture and cerebrospinal fluid leakage occurred, and the drainage tube was removed after 1 week of 
prolonged drainage. One week after the operation, the effect of scoliosis correction was acceptable, and the internal fixation was stable. Three months after the operation, the position of the intervertebral disc fusion cage was intact, the fusion was good, and the internal fixation was not loose; broken screw, broken rod, and any other complications were not observed (Figure 4).

\section{Clinical evaluation}

The ODI score was $37.25 \pm 18.54$ preoperatively and $12.45 \pm 5.64$ at 3 months postoperation; the JOA score was $15.21 \pm 2.38$ preoperatively and $23.84 \pm 1.35$ at 3 months postoperation; the VAS-BP score was $5.43 \pm 1.43$ preoperatively and $2.18 \pm 1.68$ at 3 months postoperation; and the VAS-LP score was $6.23 \pm 1.05$ preoperatively and $2.13 \pm$ 1.06 at 3 months postoperation (Table 3). The preoperative mean Cobb angle was $25.64^{\circ} \pm$ $5.38^{\circ}$, and the postoperative mean Cobb angle was $7.34^{\circ} \pm 2.39^{\circ}$; the preoperative $\mathrm{LL}$ angle was $-36.21^{\circ} \pm 11.85^{\circ}$, and the postoperative $\mathrm{LL}$ angle was $-43.54^{\circ} \pm 9.85^{\circ}$; the preoperative SS angle was $26.48^{\circ} \pm 7.98^{\circ}$, and the postoperative SS angle was $33.76^{\circ} \pm 8.14^{\circ}$; the preoperative PT angle was $21.26^{\circ} \pm 6.29^{\circ}$, and the postoperative PT angle was $14.95^{\circ} \pm$ $7.16^{\circ}$. The surgical correction rate was good. The results are shown in Table 4.

\section{Discussion}

Degenerative scoliosis is mainly caused by the degeneration of the intervertebral disc, facet joint, and other lumbar structures. It can cause low back pain and lower extremity nerve root symptoms [15]. At present, the diagnostic criterion for scoliosis is that the Cobb angle of coronal scoliosis on $\mathrm{X}$-ray should be greater than $10^{\circ}$ [16]. For lower extremity radiculopathy caused by scoliosis, we usually determine the involved nerve roots according to the pain distribution area, neurological examination, and neurography [17]. However, in our clinical work, lumbar spinal stenosis caused by scoliosis was often found to be multisegmental; moreover, some cases of nerve signs do not show typical, fuzzy positioning, which leads to difficulties in the selection of surgical decompression segment [3]. Because normal MRI can only select the level of the intervertebral disc for plain scan, and for scoliosis, the spine often shows three-dimensional deformity, the plane selected by normal MRI cannot be completely accurate. Clinical studies with conventional lumbar MRI examination have shown that the addition of the coronal sequence can more accurately determine the responsible nerve root [4]. In patients with multisegmental lumbar stenosis, the accurate selection of segmental decompression and a smaller surgical method can improve the surgical effect [11]. Recently, 
Yan Liang et al. [13] used a nerve root block to accurately locate the responsible segment and decompress the corresponding segment, and satisfactory surgical results were obtained for the patients. In the present study, we used DTI to accurately locate the responsible nerve root and to study its effectiveness and accuracy.

In this study, DTI was performed to locate the responsible segment before operation. Previous studies have shown that the FA value of symptomatic nerve roots is significantly lower than that of asymptomatic nerve roots in DTI examination, which can be used as the basis for identifying symptomatic nerve roots $[18,19]$. DTT can reconstruct the nerve into a threedimensional image, which can clearly show the compressed part of the nerve, and the abnormal segment of the image is correlated with the FA value $[19,20]$. In another study on the removal of transforaminal endoscopic nucleus pulposus, preoperative and postoperative DTI examination showed that the FA value strong correlates with the preoperative and postoperative clinical score of patients [10]. According to the results of DTI, there were 6 cases of $L 3$ nerve ( 4 left and 2 right), 18 cases of $L 4$ nerve ( 9 left and 9 right), 25 cases of $L 5$ nerve (15 left and 12 right), and 11 cases of S1 nerve (5 left and 7 right). Moreover, there were 13 cases of single segment, 21 cases of double segment, and 2 cases of three segment. We also located the responsible nerve root according to the symptoms and signs or nerve root block before operation. The results from both diagnostic methods were consistent. According to the results of DTT reconstruction, the nerve defect or sparse part was diagnosed as the responsible nerve root, and the results agreed with those of the previous two methods. Thus, our study can conclude that DTI examination can identify the responsible nerve root and guide the choice of surgical treatment.

The surgical treatment of scoliosis mainly includes two aspects. First, by correcting scoliosis deformity and recovering the normal sequence of the spine, we can relieve low back pain; second, by performing direct or indirect decompression, we can relieve the symptoms of lower extremity root pain and intermittent claudication [21]. For scoliosis, we chose long segment fixation. Combined with the results of DTI, the decompression segment was selected. Interbody fusion was performed on the resected disc segments. After the operation, the patients underwent postoperative rehabilitation under the guidance of our department of rehabilitation until discharge. The scores of ODI, JOA, VAS-BP, and VAS-LP were determined and imaging evaluation was performed at 1 week, 3 months, and 6 months after operation. The JOA scores of all patients were significantly improved after follow-up. The image evaluation of scoliosis was significantly improved as compared to that before operation, and 
the correction effect was satisfactory. Thus, it can be concluded that DTI examination can accurately determine the responsible nerve, as satisfactory relief from postoperative symptoms was achieved according to this assessment of the responsible space decompression.

Liu Hong et al. [22] summarized that in degenerative scoliosis, L3 and L4 nerve roots were compressed on the concave side, which was caused by the intervertebral foramen or intervertebral foramen stenosis, while L5 and S1 nerve roots were compressed on the convex side, which was caused by lateral recess stenosis. In this experiment, myelography was used to determine the position of compression, which is a relatively invasive method of examination. In the present study, DTI scan using a continuous T2 sequence imaging was used, which is different from conventional MRI scan only at the level of the intervertebral disc. Twodimensional reconstruction can be performed through continuous images. By adjusting the coronal plane and the sagittal plane, the complete nerve root canal can be selected in order to more accurately determine the position of nerve compression (Fig. 1). During the orthopedic process, the concave side can be opened and the nerve root canal can be subsequently opened, which enables to achieve indirect decompression. If the nerve root symptoms appear in the convex side, we need to apply pressure during the orthopedic process as the nerve root canal is relatively small. In this case, we need to achieve full decompression. Therefore, the location of symptomatic nerve roots is particularly important. In conclusion, in the process of diagnosis and treatment of patients with degenerative scoliosis, on the basis of clinical manifestations and signs of patients and combined with DTI results, we can more accurately identify the symptomatic nerve root, enabling us to more accurately select the decompression segment and location. Accurately defining the responsible segment can reduce preventive decompression, reduce the operation time, and provide a new auxiliary diagnostic method for scoliosis correction. DTI, however, has its limitations. For example, in the presence of internal fixation after spinal surgery, the FA value of DTI is affected; thus, it is not possible to conduct DTI examination for comparing neurological function, mainly through symptoms and physical examination. Therefore, DTI is mainly used for preoperative symptomatic nerve root localization.

\section{Abbreviations}

CT: computed tomography; MRI: magnetic resonance imaging; DTI: Diffusion tensor imaging; ODI, oswestry disability index; JOA, Japanese Orthopaedic Association; VAS-BP, 
visual analog scale-back pain; VAS-LP, visual analog scale-leg pain; LL, lumbar lordosis; PT pelvic tilt; SS, sacrum slope

\section{Declarations}

\section{Ethics approval and consent to participate}

The study protocol was approved by the Ethics Committee of Jiangsu Provincial Hospital and written informed consent were obtained from all patients. All methods were performed in accordance with the relevant guidelines and regulations.

\section{Consent for publication}

Not applicable.

\section{Availability of data and materials}

The data which analyzed during the study are stored in our hospital system and are available from the corresponding author on reasonable request.

\section{Competing interests}

The authors declare that they have no competing interests.

\section{Funding}

This study was supported by grants from the National Natural Science Foundation of China (81772351and 81902211). These fundings were used for data collection, analysis and publication of the article.

\section{Authors' contributions}

QW and PG carried out the acquisition and interpretation of data. PT, JC and SZ carried out the clinical data collection. QL and WZ carried out the operation and radiological measurements. GY and JF carried out the design of this study. QW and PG did the revision of the manuscript. All authors have read and approved the final manuscript.

\section{Acknowledgments}

The authors thank all colleagues who assisted in this study.

\section{References}

1. Jimbo S, Kobayashi T, Aono K, Atsuta Y, Matsuno T: Epidemiology of degenerative lumbar scoliosis: a community-based cohort study. Spine (Phila Pa 1976) 2012, 37(20):1763-1770.

2. Transfeldt EE, Topp R, Mehbod AA, Winter RB: Surgical outcomes of decompression, decompression with limited fusion, and decompression with full curve fusion for degenerative scoliosis with radiculopathy. Spine (Phila Pa 1976) 2010, 35(20):18721875.

3. Botwin KP, Gruber RD, Bouchlas CG, Torres-Ramos FM, Sanelli JT, Freeman ED, Slaten WK, Rao S: Fluoroscopically guided lumbar transformational epidural steroid injections in degenerative lumbar stenosis: an outcome study. Am J Phys Med Rehabil 2002, 81(12):898-905.

4. Lee SK, Jung JY: Degenerative lumbar scoliosis: added value of coronal images to routine lumbar MRI for nerve root compromise. Eur Radio/2020, 30(4):2270-2279.

5. Chazen JL, Cornman-Homonoff J, Zhao Y, Sein M, Feuer N: MR Neurography of the 
Lumbosacral Plexus for Lower Extremity Radiculopathy: Frequency of Findings, Characteristics of Abnormal Intraneural Signal, and Correlation with Electromyography. AJNR Am J Neuroradio/ 2018, 39(11):2154-2160.

6. Oikawa Y, Eguchi Y, Inoue G, Yamauchi K, Orita S, Kamoda H, Ishikawa T, Miyagi M, Suzuki $M$, Sakuma $Y$ et al: Diffusion tensor imaging of lumbar spinal nerve in subjects with degenerative lumbar disorders. Magn Reson Imaging 2015, 33(8):956-961.

7. Cauley KA, Filippi CG: Diffusion-tensor imaging of small nerve bundles: cranial nerves, peripheral nerves, distal spinal cord, and lumbar nerve roots--clinical applications. AJR Am J Roentgeno/ 2013, 201(2):W326-335.

8. Shi Y, Zou Y, Feng Y, Dou W, Ding H, Wang C, Zhao F, Shi H: A quantitative and clinical evaluation of nerve roots in lumbosacral radiculopathy using diffusion tensor imaging. Jpn J Radio/ 2020, 38(3):222-230.

9. Eguchi Y, Oikawa Y, Suzuki M, Orita S, Yamauchi K, Suzuki M, Aoki Y, Watanabe A, Takahashi K, Ohtori S: Diffusion tensor imaging of radiculopathy in patients with lumbar disc herniation: preliminary results. Bone Joint J2016, 98-B(3):387-394.

10. Shi Y, Zhao F, Dou W, Ding H, Zou Y, Feng Y, Wang C, Shi H, Ren Y: Quantitative Evaluation of Intraspinal Lumbar Disc Herniation-related Lumbosacral Radiculopathy Before and After Percutaneous Transforaminal Endoscopic Discectomy Using Diffusion Tensor Imaging. Spine (Phila Pa 1976) 2021, Publish Ahead of Print.

11. Shabat S, Arinzon Z, Folman Y, Leitner J, David R, Pevzner E, Gepstein R, Pekarsky I, Shuval I: Long-term outcome of decompressive surgery for lumbar spinal stenosis in octogenarians. Eur Spine J 2008, 17(2):193-198.

12. Osunronbi T, Sharma H: International Standards for Neurological Classification of Spinal Cord Injury: factors influencing the frequency, completion and accuracy of documentation of neurology for patients with traumatic spinal cord injuries. Eur J Orthop Surg Traumato/ 2019, 29(8):1639-1648.

13. Liang Y, Zhao Y, Wang T, Zhu Z, Liu H, Mao K: Precision Treatment of Adult Lumbar Degenerative Scoliosis Complicated by Lumbar Stenosis with the Use of Selective Nerve Root Block. World Neurosurg 2018, 120:e970-e975.

14. Shi $Y$, Zong $M, X u X$, Zou $Y$, Feng $Y$, Liu W, Wang C, Wang D: Diffusion tensor imaging with quantitative evaluation and fiber tractography of lumbar nerve roots in sciatica. Eur J Radio/ 2015, 84(4):690-695.

15. Graham RB, Sugrue PA, Koski TR: Adult Degenerative Scoliosis. Clin Spine Surg 2016, 29(3):95-107.

16. Ploumis A, Transfledt EE, Denis F: Degenerative lumbar scoliosis associated with spinal stenosis. Spine J 2007, 7(4):428-436.

17. Fu KM, Rhagavan P, Shaffrey $\mathrm{Cl}$, Chernavvsky DR, Smith JS: Prevalence, severity, and impact of foraminal and canal stenosis among adults with degenerative scoliosis. Neurosurgery 2011, 69(6):1181-1187.

18. Eguchi Y, Ohtori S, Orita S, Kamoda H, Arai G, Ishikawa T, Miyagi M, Inoue G, Suzuki M, Masuda $Y$ et al: Quantitative evaluation and visualization of lumbar foraminal nerve root entrapment by using diffusion tensor imaging: preliminary results. AJNR Am J Neuroradio/ 2011, 32(10):1824-1829. 
19. Balbi V, Budzik JF, Duhamel A, Bera-Louville A, Le Thuc V, Cotten A: Tractography of lumbar nerve roots: initial results. Eur Radio/ 2011, 21(6):1153-1159.

20. Eguchi $Y$, Ohtori S, Suzuki M, Oikawa Y, Yamanaka H, Tamai H, Kobayashi T, Orita S, Yamauchi K, Suzuki M et al: Discrimination between Lumbar Intraspinal Stenosis and Foraminal Stenosis using Diffusion Tensor Imaging Parameters: Preliminary Results. Asian Spine J 2016, 10(2):327-334.

21. Zhao $Y$, Liang $Y$, Mao K: Radiographic and clinical outcomes following MIS-TLIF in patients with adult lumbar degenerative scoliosis. J Orthop Surg Res 2018, 13(1):93.

22. Liu H, Ishihara H, Kanamori M, Kawaguchi Y, Ohmori K, Kimura T: Characteristics of nerve root compression caused by degenerative lumbar spinal stenosis with scoliosis. Spine J 2003, 3(6):524-529.
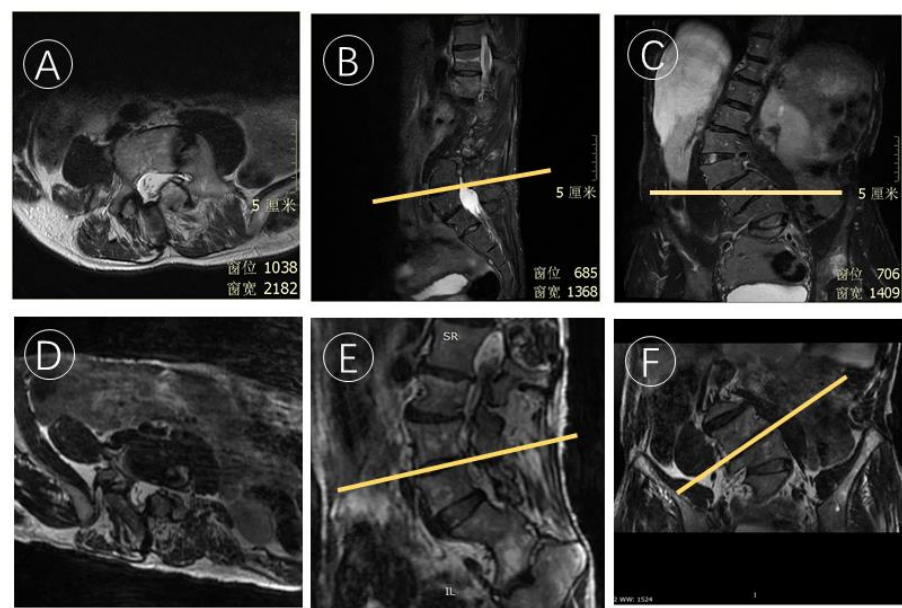

Fig. $1 \mathrm{~A}, \mathrm{~B}$ and $\mathrm{C}$ are ordinary MRI. When axial films are selected, the level of intervertebral disc is selected through the sagittal plane, but in the coronal plane, the axial film is not at the level of intervertebral disc, so the axial film has no reference value. D. E and F were continuous T2 weighted images in DTI examination. After two-dimensional reconstruction by software, the axial films were at the level of intervertebral disc in sagittal plane and coronal plane.
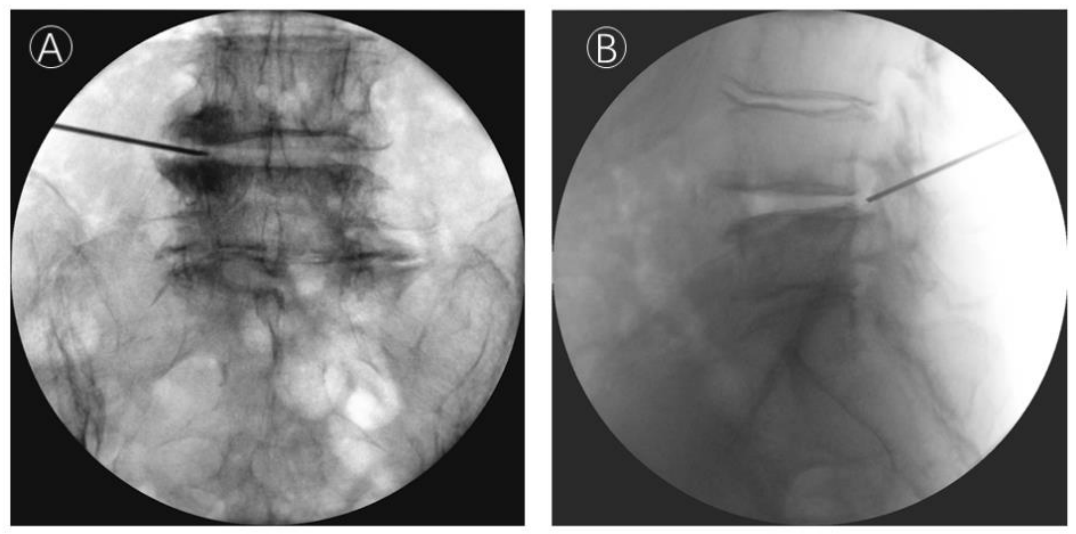

Fig.2 Intraoperative X-ray of anteroposterior and lateral position for the nerve root block operation. 

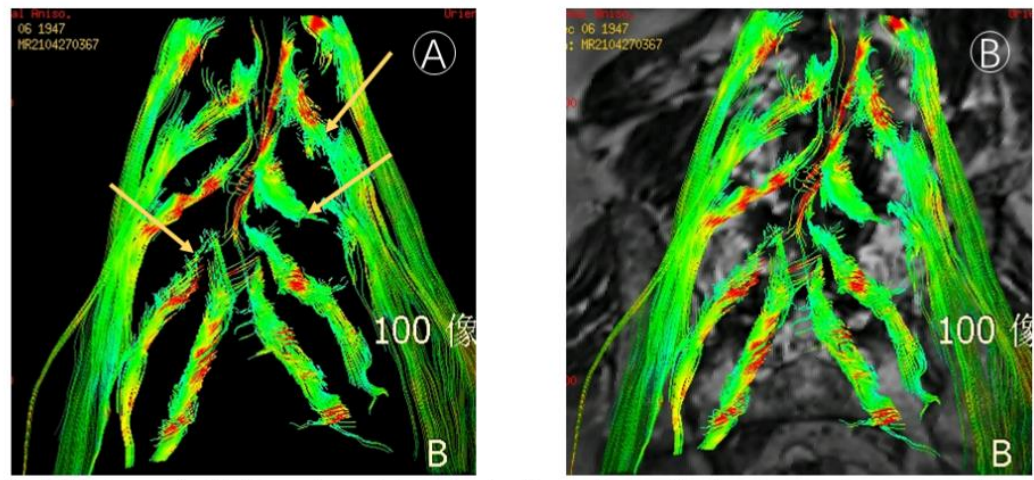

Fig. 3. $A$ is the DTT image in DTI imaging, which clearly shows the entire lumbosacral nerve root. The position indicated by the arrow shows that the nerve root is sparse, which is the responsible nerve root. This finding is consistent with the result of nerve root block. B is the projection of DTT image on the lumbar spine.
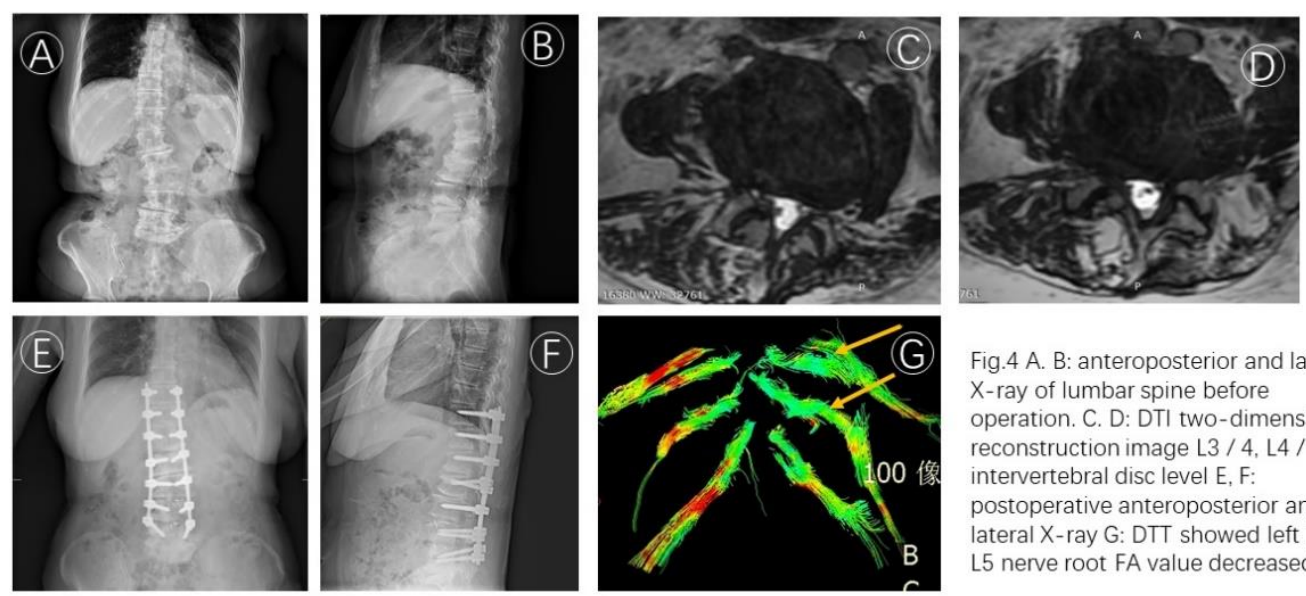

Fig.4 A. B: anteroposterior and lateral $X$-ray of lumbar spine before operation. C. D: DTI two-dimensional

reconstruction image L3 / 4, L4 / 5

intervertebral disc level E, F:

postoperative anteroposterior and lateral X-ray G: DTT showed left L4

$L 5$ nerve root $F A$ value decreased.

Table 1 Clinical and DTI results of symptomatic nerve roots

\begin{tabular}{ccccc}
\hline & Clinical & DTI & Nerve root block & DTI \\
\hline single segment & 5 & 5 & 8 & 8 \\
double segments & 7 & 7 & 14 & 14 \\
three segments & 1 & 1 & 1 & 1 \\
L3 L & 3 & 3 & 1 & 1 \\
L4 L & 3 & 3 & 7 & 6 \\
L5 L & 4 & 4 & 12 & 11 \\
S1 L & 3 & 2 & 3 & 3 \\
L3 R & 1 & 1 & 1 & 1 \\
L4 R & 2 & 2 & 10 & 7 \\
L5 R & 3 & 3 & 3 & 3 \\
S1 R & 4 & 4 & & 9 \\
\hline
\end{tabular}


Table 2 Comparison of FA values of symptomatic and asymptomatic nerve roots

\begin{tabular}{ccccc}
\hline \multirow{2}{*}{ segment } & \multicolumn{5}{c}{ FA value } & & \\
\cline { 2 - 5 } & symptomatic & asymptomatic & P-value & Significance \\
\hline L3 & $0.162 \pm 0.026$ & $0.231 \pm 0.031$ & 0.0035 & $* *$ \\
L4 & $0.145 \pm 0.024$ & $0.254 \pm 0.032$ & 0.0024 & $* *$ \\
L5 & $0.152 \pm 0.033$ & $0.237 \pm 0.024$ & 0.0014 & $* *$ \\
S1 & $0.132 \pm 0.021$ & $0.201 \pm 0.034$ & 0.0017 & $*$ \\
\hline
\end{tabular}

Note: Data are shown as mean \pm standard deviation. $\mathrm{P}<0.01$, significant difference. Independent samples t-test.

Table 3 Comparison of ODI, JOA, VAS-BP, and VAS-LP scores of patients at pre- and postoperation

\begin{tabular}{ccccc}
\hline & pre-operation & 3month & 6 months & Last time \\
\hline ODI scores & $37.25 \pm 18.54$ & $12.45 \pm 5.64^{* *}$ & $10.75 \pm 3.36^{\star *}$ & $9.86 \pm 2.35^{\star *}$ \\
JOA scores & $15.21 \pm 2.38$ & $23.84 \pm 1.35^{\star *}$ & $25.34 \pm 2.25^{\star *}$ & $25.52 \pm 1.84^{\star *}$ \\
VAS-BP & $5.43 \pm 1.43$ & $2.18 \pm 1.68^{* *}$ & $1.98 \pm 0.85^{* *}$ & $1.87 \pm 0.96^{\star *}$ \\
VAS-LP & $6.23 \pm 1.05$ & $2.13 \pm 1.06^{* *}$ & $1.96 \pm 0.76^{* *}$ & $1.94 \pm 0.65^{\star *}$ \\
\hline
\end{tabular}

Note: Data are shown as mean \pm standard deviation. ${ }^{*} \mathrm{P}<0.01$, significant difference. Independent samples t-test.

ODI, Oswestry Disability Index; JOA, Japanese Orthopaedic Association; VAS-BP, Visual Analog Scale-Back Pain; VAS-LP, Visual Analog Scale-Leg Pain

Table 4 Radiologic assessments of patients at pre- and postoperation

\begin{tabular}{llll}
\hline pre-operation 3 3month & $p$-value & Significance \\
\hline
\end{tabular}




$\begin{array}{crrrr}\text { Cobb, }{ }^{\circ} & 25.64^{\circ} \pm 5.38^{\circ} & 7.34^{\circ} \pm 2.39^{\circ} & 0.0024 & \text { ** } \\ \text { LL, }^{\circ} & -36.21 \pm 11.85 & -43.54 \pm 9.85 & 0.0074 & \text { ** } \\ \text { SS, } & & & \\ & 26.48 \pm 7.98 & 33.76 \pm 8.14 & 0.0049 & * * \\ \text { PT, } & & & & \\ & 21.26 \pm 6.29 & 14.95 \pm 7.16 & 0.0019\end{array}$ **

Note: Data are shown as mean \pm standard deviation. ${ }^{* \star} \mathrm{P}<0.01$, significant difference. Independent samples t-test.

LL, Lumbar Lordosis; PT, Pelvic Tilt; SS, Sacral Slope 


\section{Figures}
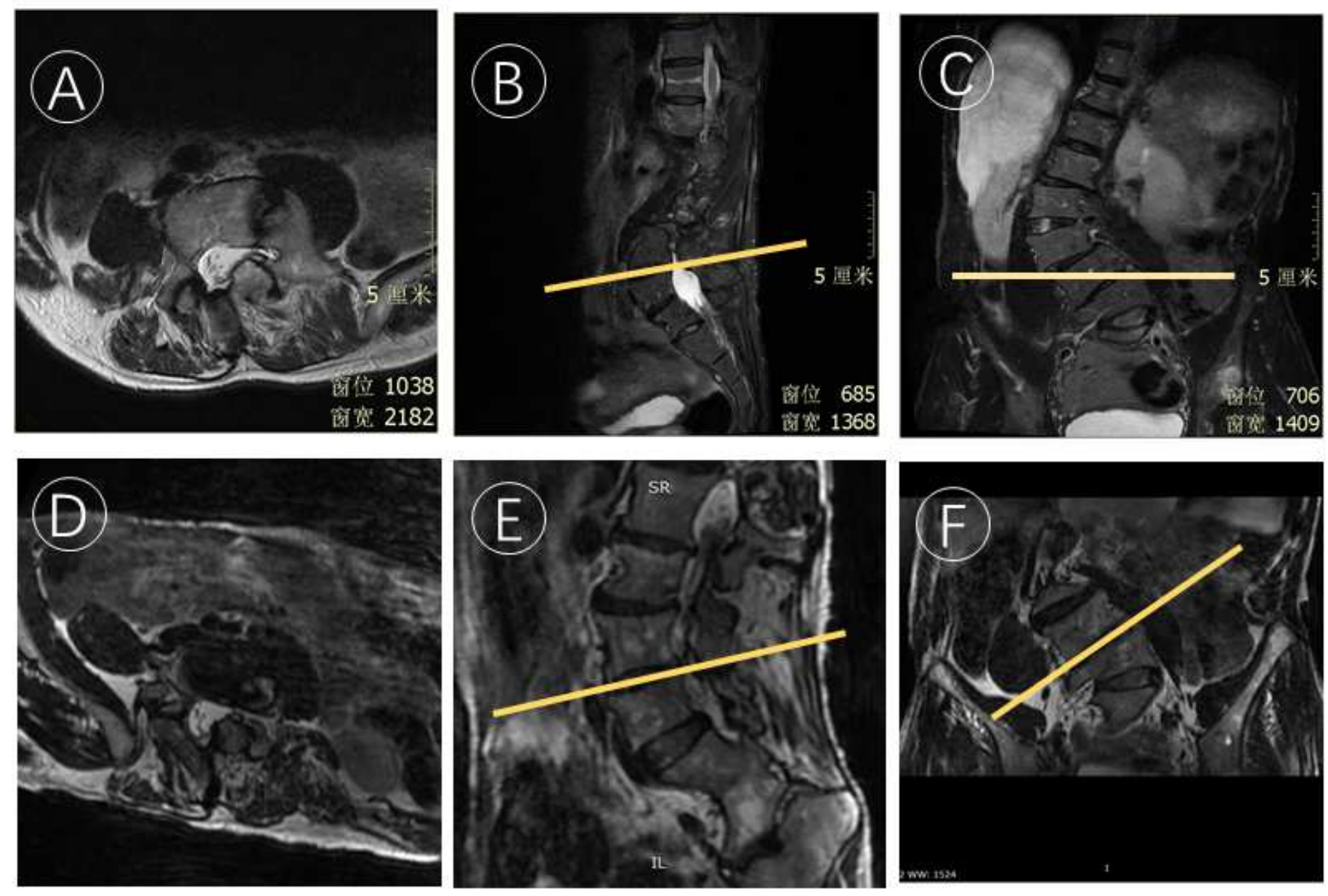

\section{Figure 1}

A, B and C are ordinary MRI. When axial films are selected, the level of intervertebral disc is selected through the sagittal plane, but in the corona! plane, the axial film is not at the level of intervertebral disc, so the axial film has no reference value. D. E and F were continuous T2 weighted images in DTI examination. Aftertwo-dimensional reconstruction by software, the axial films were at the level of intervertebral disc in sagittal plane and corona! plane. 

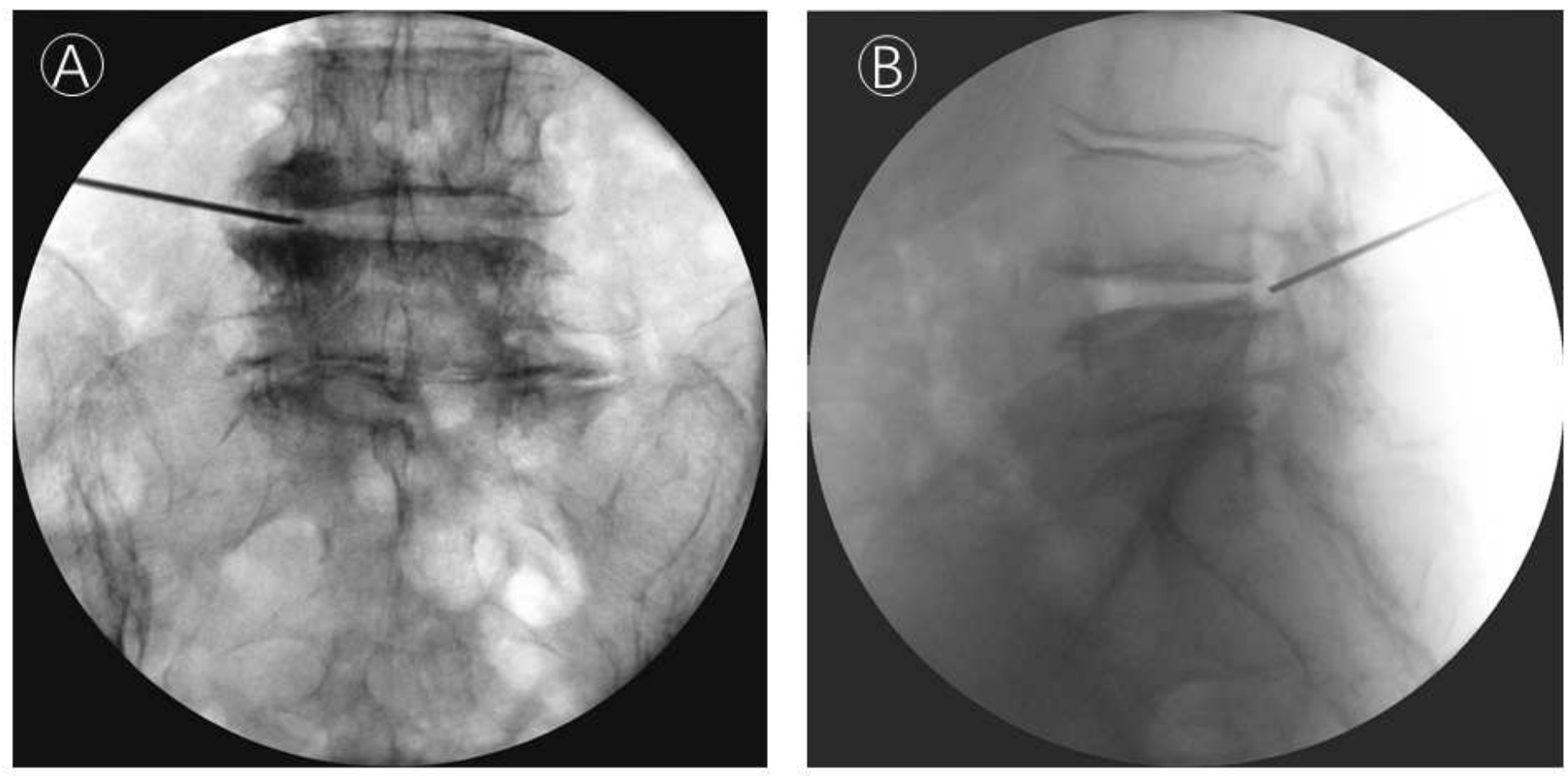

Figure 2

Intraoperative X-ray of anteroposterior and lateral position for the nerve root block operation.
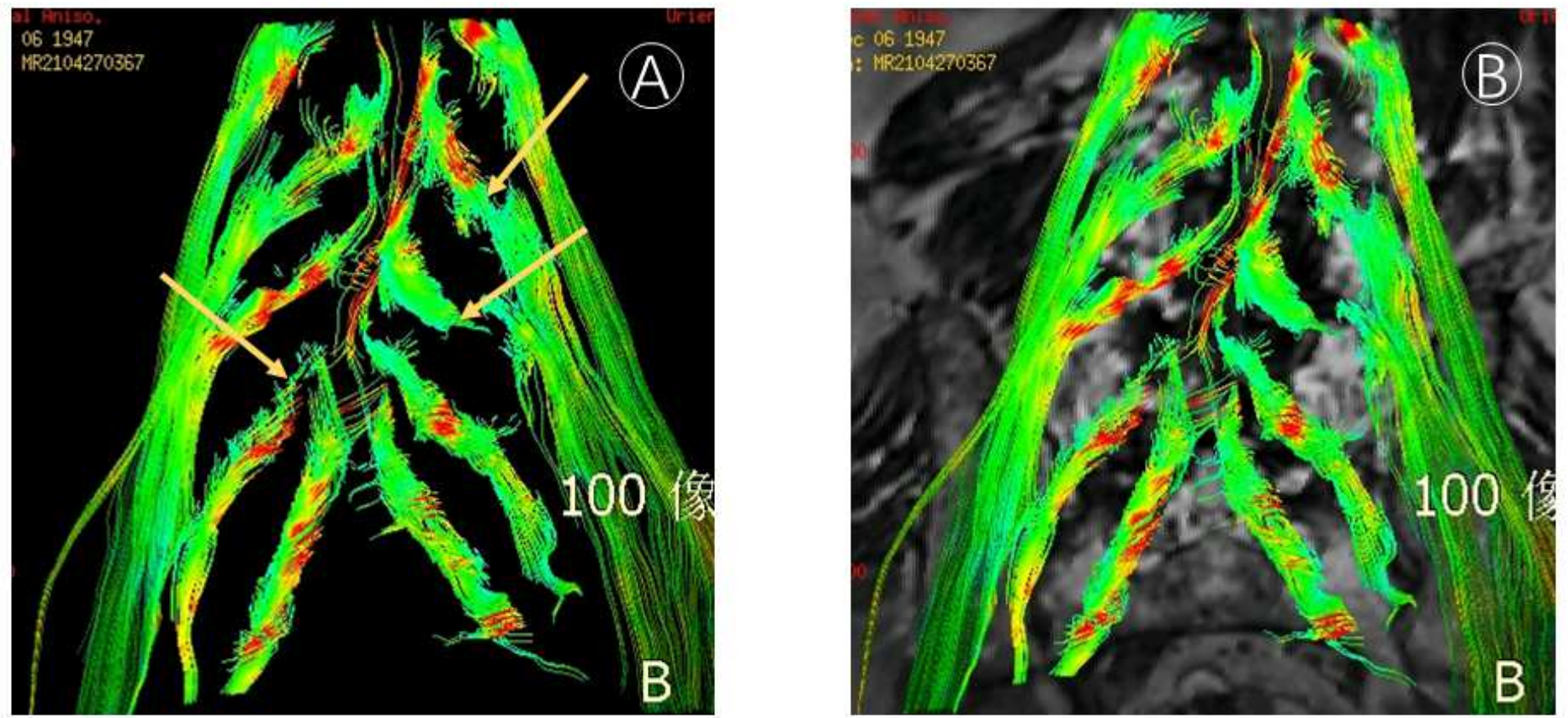

Figure 3

A is the DU image in DTI imaging, which clearly shows the entire lumbosacral nerve root. The position indicated by the arrow shows that the nerve root is sparse, which is the responsible nerve root. This 
finding is consistent with the result of nerve root block B is the projection of DTI image on the lumbar spine.
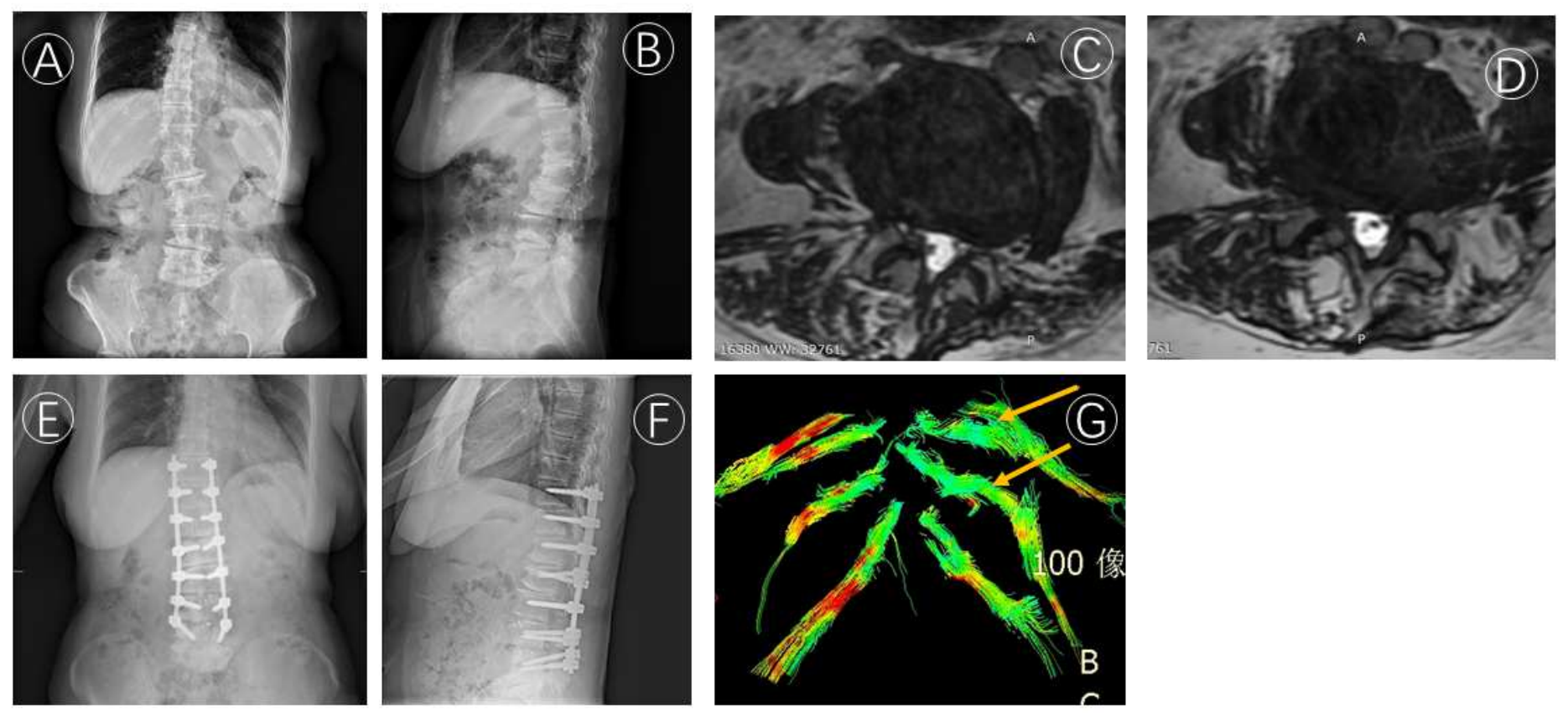

Figure 4

A. antemoostertor a. lateral X-ray of lumbar so,ne before operation. C. D: on two-Dimensional reconstruction image L3 / 4. L4 / 5 mterverteloral Disc level E, F: postoperative anteropostenor and lateral X-ray G: DTT showed left L4. L5 nerve root EA value decreased.

\section{Supplementary Files}

This is a list of supplementary files associated with this preprint. Click to download.

- Table.pdf 